Y. Teranishi

Nagoya Math. J.

Vol. 121 (1991), 149-159

\title{
EXPLICIT DESCRIPTIONS OF TRACE RINGS OF GENERIC 2 BY 2 MATRICES
}

\author{
YASUO TERANISHI
}

\section{§1. Introduction}

Let $K$ be a field of characteristic zero and let

$$
X_{1}=\left(x_{i j}(1)\right), \cdots, X_{m}=\left(x_{i j}(m)\right), \quad m \geq 2,
$$

be $m$ generic $n$ by $n$ matrices over $K$. That is, $x_{i j}(k)$ are independent commuting indeterminates over $K$. The $K$-subalgebra generated by $X_{1}, \cdots, X_{m}$ is called a ring of $n$ by $n$ generic matrices and is denoted by $R(n, m)$. Let $M_{n}\left(K\left[x_{i j}(k)\right]\right)$ denote the $n$ by $n$ matrix algebra over the polynomial ring $K\left[x_{i j}(k)\right]$. The ring $R(n, m)$ is a $K$-subalgebra of $M_{n}\left(K\left[x_{i j}(k)\right]\right)$. Let $C(n, m)$ be the subring of the polynomial ring $K\left[x_{i j}(k)\right]$ generated by all traces $\operatorname{Tr}\left(X_{i_{1}} \cdots X_{i_{\alpha}}\right)$, where $X_{i_{1}} \cdots X_{i_{\alpha}}$ is a monomial in the generic matrices $X_{1}, \cdots, X_{m}$. The trace ring $T(n, m)$ of $m$ generic $n$ by $n$ matrices is the $K$-subalgebra of $M_{n}\left(K\left[x_{i j}(k)\right]\right)$ generated by $R(n, m)$ and $C(n, m)$. Here we identify elements of $C(n, m)$ with scalar matrices.

In this paper we will be concerned with the trace ring $T(2, m)$ of generic 2 by 2 matrices. L. Le Bruyn [1. Chap. 3, Theorem 5.1] proved that $T(2, m)$ is a Cohen-Macaulay module over $C(n, m)$. Apart from this general result, very little is known about explicit structure on $T(2, m)$. Explicit descriptions of $T(2, m)$ are known only for $m \leq 4$ (cf. [2], [3], [4]) and except these cases nothing is known on an explicit description of $T(2, m)$. In this paper we will give explicit descriptions of $T(2, m)$ for all $m$.

A Young tableau on numbers $1,2, \cdots, m$

$$
Y=\left[\begin{array}{lll}
i_{1} & i_{2} \cdots i_{r} \\
j_{1} & j_{2} \cdots j_{r}
\end{array}\right]
$$

is called standard if the entries strictly increase down columns and nondecrease across rows. Let $X_{1}, \cdots, X_{m}$ be $m$ generic 2 by 2 matrices. We

Received March 22, 1990. 
denote by $\operatorname{Tr}(Y)$ the element $\operatorname{Tr}\left(X_{i_{1}} X_{j_{1}}\right) \operatorname{Tr}\left(X_{i_{2}} X_{j_{2}}\right) \cdots \operatorname{Tr}\left(X_{i_{r}} X_{j_{r}}\right)$ of $C(2, m)$. A standard monomial of $T(2, m)$ is an element of the form

$$
\operatorname{Tr}(Y) \operatorname{Tr}\left(X_{1}\right)^{\alpha_{1}} \ldots \operatorname{Tr}\left(X_{m}\right)^{\alpha_{m}} X_{1}^{\beta_{1}} \cdots X_{m}^{\beta_{m}},
$$

where $\alpha_{i}, \beta_{i}$ are non-negative integers and $Y$ is a standard tableau. We include the case that the shape of $Y$ is the empty Young diagram, and in that case we set $\operatorname{Tr}(Y)=1$. An $S$-standard monomial of $T(2, m)$ is an element of the form

$$
\operatorname{Tr}(Y) X_{i_{1}} X_{i_{2}} \cdots X_{i_{k}}
$$

where $1 \leq i_{1}<i_{2}<\cdots<i_{k} \leq m, k \geq 0$, and $Y$ is an $S$-standard Young tableau. Here if $k=0$, we set $X_{i_{1}} X_{i_{2}} \cdots X_{i_{k}}=1$. The definition of an $S$-standard Young tableau is given in the next section. Let $p_{3}, \cdots, p_{2 m-1}$ be the elements of $C(2, m)$ defined by

$$
p_{k}=\sum_{i+j=k} \operatorname{Tr}\left(X_{i} X_{j}\right), \quad 3 \leq k \leq 2 m-1,
$$

and denote by $B(2, m)$ the subring of $C(2, m)$ generated by

$$
\operatorname{Tr}\left(X_{i}\right), \quad \operatorname{Tr}\left(X_{i}^{2}\right), \quad 1 \leq i \leq m, \quad \text { and } \quad p_{k}, \quad 3 \leq k \leq 2 m-1 .
$$

Then it can be easily verified that the elements above are algebraically independent over $K$ and hence $B(2, m)$ is a polynomial ring.

C. Procesi [5] founded a $K$-basis for $T(2, m)$. The following theorem gives a natural $K$-basis for $T(2, m)$ :

THEOREM 1. The set of standard monomials of $T(2, m)$ is a $K$-basis of $T(2, m)$ over the polynomial ring $B(2, m)$.

The main result of this paper is the following

THEOREM 2. The set of S-standard monomials of $T(2, m)$ is a basis of $T(2, m)$ over the polynomial ring $B(2, m)$.

\section{§2. S-standard Young tableaux}

Consider the finite subset $\Lambda_{m}$ of $N^{2}$ :

$$
\Lambda_{m}=\left\{(i, j) \in N^{2} \mid 1 \leq i<j \leq m\right\} .
$$

The set $\Lambda_{m}$ is a partially ordered set by defining

$$
(i, j) \leq(k, l) \Leftrightarrow i \leq k \text { and } j \leq l .
$$

We denote the Hasse diagram associated with the pratially ordered set 
$\Lambda_{m}$ also by $\Lambda_{m}$, and assign to every edge in $\Lambda_{m}$ a natural number according to the following rule:

$$
\mu((i, j),(i, j+1))=2(j-1)
$$

and

$$
\mu((i, j),(i+1, j))=2 i+1 .
$$

Moreover we assign to each maximal chain in $\Lambda_{m}$

$$
(1,2)=\left(i_{0}, j_{0}\right)<\left(i_{1}, j_{1}\right)<\cdots<\left(i_{2 m-4}, j_{2 m-4}\right)=(m-1, m)
$$

a standard Young tableau

$$
\left[\begin{array}{lll}
\cdots & i_{\alpha} \cdots \\
\cdots & j_{\alpha} & \cdots
\end{array}\right] \quad \alpha \in S
$$

where $S$ is the subset of indices $\alpha \in\{0,1, \cdots, 2 m-4\}$ such that

$$
\mu\left(\left(i_{\alpha-1}, j_{\alpha-1}\right),\left(i_{\alpha}, j_{\alpha}\right)\right)>\mu\left(\left(i_{\alpha}, j_{\alpha}\right),\left(i_{\alpha+1}, j_{\alpha+1}\right)\right) .
$$

We call a standard tableau, obtained as above, an $S$-standard tableau.

\section{§3. Grassmannian Gr $(2, m)$ and Procesi's identity}

Let $\operatorname{Gr}(2, m)$ be the Grassmannian of the 2-dimensional $K$-vector spaces of an $m$-dimensional fixed $K$-vector space. The homogeneous coordinate ring $K[\operatorname{Gr}(2, m)]$ of $\operatorname{Gr}(2, m)$ is generated by the Prücker coordinates $p_{i j}, 1 \leq i<j \leq m$. A monomial in the Prücker coordinates

$$
p_{i_{1} j_{1}} p_{i_{2} j_{2}} \cdots p_{i_{r} j_{r}}
$$

is called a standard monomial if the associated Young tableau

$$
\left[\begin{array}{lll}
i_{1} i_{2} & \cdots & i_{r} \\
j_{1} j_{2} & \cdots & j_{r}
\end{array}\right]
$$

is standard. Let

$$
\theta_{k}=\sum_{i+j=k} p_{i j}, \quad \text { for } k=3,4, \cdots, 2 m-1 .
$$

Then $\theta_{3}, \theta_{4}, \cdots, \theta_{2 m-1}$ are algebraically independent over $K$. We now recall the following basic results on the homogeneous coordinate ring of the Grassmannian $\operatorname{Gr}(2, m)$.

Proposition 1 (cf. [6]). The set of standard monomials is a K-basis of the homogeneous coordinate ring $K[\mathrm{Gr}(2, m)]$. 
Proposition 2 (cf. [7]). The homogeneous coordinate ring $K[\mathrm{Gr}(2, m)]$ is a free module of finite rank over the polynomial ring $K\left[\theta_{3}, \theta_{4}, \cdots, \theta_{2 m-1}\right]$ and the set of standard monomials associated with $S$-standard tableaux is $a$ basis of $K[\mathrm{Gr}(2, m)]$ over $K\left[\theta_{3}, \theta_{4}, \cdots, \theta_{2 m-1}\right]$.

We make $K[\operatorname{Gr}(2, m)]$ into a graded ring by giving each $p_{i j}$ degree 2. Denoting by $K[\operatorname{Gr}(2, m)]_{d}$ the $K$-vector space of degree $d$-part, we consider the Poincare series associated with $K[\operatorname{Gr}(2, m)]$ :

$$
P(K[\operatorname{Gr}(2, m)], t)=\sum_{d \geq 0} \operatorname{dim} K\{\operatorname{Gr}(2, m)]_{d} t^{d} .
$$

By Proposition 1, we have

$$
P(K[\operatorname{Gr}(2, m)], t)=\sum_{d \geq 0} \sharp\left\{\begin{array}{l}
\text { standard monomials of } \\
K[\operatorname{Gr}(2, m)] \text { with degree } d
\end{array}\right\} t^{d} .
$$

The trace ring $T(2, m)$ of $m$ generic 2 by 2 matrices is also a graded ring by giving each $x_{i j}(k)$ degree 1 . Denoting by $T(2, m)_{d}$ the $K$-vector space of $T(2, m)$ spanned by all homogeneous elements of degree $d$, we consider the Poincare series of $T(2, m)$ :

$$
P(T(2, m), t)=\sum_{d \geq 0} \operatorname{dim} T(2, m)_{d} t^{d} .
$$

C. Procesi discovered the following identity between $P(T(2, m), t)$ and $P(K[\operatorname{Gr}(2, m), t)$ :

Proposition 3 (C. Procesi).

$$
P(T(2, m), t)=(1-t)^{-2 m} P(K[\mathrm{Gr}(2, m)], t) .
$$

For the proof we refer the reader to [1. Chap. 5] or [4, Proposition 8.1]. Procesi used a sort of Pieri's formula. A direct proof is given in [4].

\section{§4. The Streightening formula}

In this section we will prove Theorem 1 . Let $X_{1}, \cdots, X_{m}$ be $m$ generic 2 by 2 matrices. The matrices $X_{1}^{0}, \cdots, X_{m}^{0}$ defined by

$$
X_{i}^{0}=X_{i}-\frac{1}{2} \operatorname{Tr}\left(X_{i}\right), \quad \text { for } i=1, \cdots, m,
$$

are called 2 by 2 generic trace zero matrices. The $K$-subalgebra of $T(2, m)$ generated by $X_{1}^{0}, \cdots, X_{m}^{0}$ and all traces of the monomials in $X_{\imath}^{0}$, $1 \leq i \leq m$, is called the ring of $m$ generic 2 by 2 trace zero matrices, 
and will be denoted by $T^{0}(2, m)$. The trace ring $T(2, m)$ is clearly a polynomial ring over $T^{0}(2, m)$ :

$$
T(2, m)=T^{0}(2, m)\left[\operatorname{Tr}\left(X_{1}\right), \cdots, \operatorname{Tr}\left(X_{m}\right)\right] .
$$

By using the Cayley-Hamilton formula for 2 by 2 matrices, it can be easily shown that $T^{0}(2, m)$ is generated by $X_{1}^{0}, \cdots, X_{m}^{0}$ and they satisfy the following relation:

$$
X_{i}^{0} X_{j}^{0}+X_{j}^{0} X_{i}^{0}=\operatorname{Tr}\left(X_{i}^{0} X_{j}^{0}\right), \quad \text { for all } i, j .
$$

Using the relation (4.2), we see that any element of $T^{0}(2, m)$ is a $K$-linear combination of monomials of the form

$$
\begin{aligned}
& \operatorname{Tr}\left(X_{i_{1}}^{0} X_{j_{1}}^{0}\right) \cdots \operatorname{Tr}\left(X_{i_{r}}^{0} X_{j_{r}}^{0}\right) X_{k_{1}}^{0} \cdots X_{k t}^{0}, \\
& \quad 1 \leq i_{\alpha} \leq j_{\alpha} \leq m, \quad 1 \leq k_{\beta} \leq m, \quad \text { and } \quad r, t \geq 0
\end{aligned}
$$

We call $X_{k_{1}}^{0} \cdots X_{k_{t}}^{0}$ the matrix part and $\operatorname{Tr}\left(X_{i_{1}}^{0} X_{j_{1}}^{0}\right) \cdots \operatorname{Tr}\left(X_{i_{r}}^{0} X_{j_{r}}^{0}\right)$ the trace part. If $t=0$ (resp. $r=0$ ), we set

$$
X_{k_{1}}^{0} \cdots X_{k_{t}}^{0} \quad\left(\text { resp. } \operatorname{Tr}\left(X_{i_{1}}^{0} X_{j_{1}}^{0}\right) \cdots \operatorname{Tr}\left(X_{i_{r}}^{0} X_{j_{r}}^{0}\right)=1\right) .
$$

Using the relation (4.2) again, we can normalize the matrix part of (4.3) into regular order. Therefore any element of $T^{0}(2, m)$ is a $K$-linear combination of monomials of the form

$$
\operatorname{Tr}\left(X_{i_{1}}^{0} X_{j_{1}}^{0}\right) \operatorname{Tr}\left(X_{i_{2}}^{0} X_{j_{2}}^{0}\right) \cdots \operatorname{Tr}\left(X_{i_{r}}^{0} X_{j_{r}}^{0}\right)\left(X_{1}^{0}\right)^{\alpha_{1}} \cdots\left(X_{m}^{0}\right)^{\alpha_{m}}
$$

with $\alpha_{i} \in \mathbf{N}, i_{\alpha}<j_{\alpha}$, for all $\alpha$, and $1 \leq i_{1} \leq i_{2} \leq \cdots \leq i_{r}$. Such a monomial is called a semi-standard monomial, and a semi-standard monomial is called a standard monomial if the Young tableau associated with its trace part is a standard tableau.

Proof of Theorem 1. First, we prove that any semi-standard monomial of $T^{0}(2, m)$ is a $K$-linear combination of standard monomials. Take a semi-standard monomial (4.4) with degree $d$ and let

$$
\underline{a}=(\underbrace{\cdots \cdots 1}_{\alpha_{1}}, \underbrace{2 \cdots 2}_{\alpha_{2}}, \cdots, \underbrace{m \cdots m}_{\alpha_{m}}) .
$$

We insert the numbers $i_{1}, j_{1}, i_{2}, j_{2}, \cdots, i_{r}, j_{r}$ into the sequence $\underline{a}$ as follows: if $i_{1}=\cdots=i_{k}<i_{k+1}$ for some $k$, insert the numbers $i_{1}, j_{1}, \cdots, i_{k}, j_{k}$ into $\underline{a}$ by means the rule below and we get a sequence $\underline{a}\left[i_{1} j_{1} \cdots i_{k} j_{k}\right]$ of numbers; 


$$
\underline{a}\left[i_{1} j_{1} \cdots i_{k} j_{k}\right]=(\cdots, \underbrace{i_{1} \cdots i_{1}}_{\alpha_{i_{1}}}, i_{1} j_{1} \cdots i_{k} j_{k}, \underbrace{i_{1}+1 \cdots i_{1}+1}_{\alpha_{i_{1}+1}}, \cdots) .
$$

Repeating this procedure successibly, we obtain a sequence of numbers $\underline{a}\left[i_{1} j_{1} \cdots i_{r} j_{r}\right] \in N^{d}$ and call it the content of $f$ (denoted by $c(f)$ ). For example, if $f=\operatorname{Tr}\left(X_{1}^{0} X_{2}^{0}\right) X_{1}^{0} X_{2}^{0}$, we have $c(f)=(1,1,2,2)$.

The following identity on 2 by 2 trace zero matrices $X_{1}, \cdots, X_{4}$ is a consequence of the Cayley-Hamilton theorem for 2 by 2 matrices.

$$
\begin{aligned}
\operatorname{Tr}\left(X_{1} X_{2}\right) & \operatorname{Tr}\left(X_{3} X_{4}\right) \\
= & \operatorname{Tr}\left(X_{1} X_{3}\right) \operatorname{Tr}\left(X_{2} X_{4}\right)-\operatorname{Tr}\left(X_{1} X_{4}\right) \operatorname{Tr}\left(X_{2} X_{3}\right)-4 X_{1} X_{2} X_{3} X_{4} \\
& +2\left\{\operatorname{Tr}\left(X_{1} X_{2}\right) X_{3} X_{4}+\operatorname{Tr}\left(X_{3} X_{4}\right) X_{1} X_{2}-\operatorname{Tr}\left(X_{1} X_{3}\right) X_{2} X_{4}\right. \\
& \left.-\operatorname{Tr}\left(X_{2} X_{4}\right) X_{1} X_{3}+\operatorname{Tr}\left(X_{1} X_{4}\right) X_{2} X_{3}+\operatorname{Tr}\left(X_{2} X_{3}\right) X_{1} X_{4}\right\}
\end{aligned}
$$

Suppose now that a semi-standard monomial (4.4) is not a standard monomial. Then there exists a number $k$ such that

$$
i_{k}<i_{k+1}<j_{k+1}<j_{k} .
$$

Then applying the identity (4.5) to $\operatorname{Tr}\left(X_{i_{k}} X_{j_{k}}\right) \operatorname{Tr}\left(X_{i_{k+1}} X_{j_{k+1}}\right)$, we obtain:

$$
\begin{aligned}
\operatorname{Tr}\left(X_{i_{k}}^{0} X_{j_{k}}^{0}\right) & \operatorname{Tr}\left(X_{i_{k+1}}^{0} X_{j_{k+1}}^{0}\right)\left(X_{1}^{0}\right)^{\alpha_{1}} \cdots\left(X_{m}^{0}\right)^{\alpha_{m}} \\
= & \operatorname{Tr}\left(X_{i_{k}}^{0} X_{i_{k+1}}^{0}\right) \operatorname{Tr}\left(X_{j_{k+1}}^{0} X_{j_{k}}^{0}\right)\left(X_{1}^{0}\right)^{\alpha_{1}} \cdots\left(X_{m}^{0}\right)^{\alpha_{m}} \\
& -\operatorname{Tr}\left(X_{i_{k}}^{0} X_{j_{k+1}}^{0}\right) \operatorname{Tr}\left(X_{i_{k+1}}^{0} X_{j_{k}}^{0}\right)\left(X_{1}^{0}\right)^{\alpha_{1}} \cdots\left(X_{m}^{0}\right)^{\alpha_{k}} \\
& -4\left(X_{1}^{0}\right)^{\alpha_{1}} \cdots\left(X_{t}^{0}\right)^{\alpha_{t}}\left(X_{i_{k}}^{0} X_{j_{k}}^{0} X_{i_{k+1}}^{0} X_{j_{k+1}}^{0}\right)\left(X_{t+1}^{0}\right)^{\alpha_{t+1}} \cdots\left(X_{m}^{0}\right)^{\alpha_{m}} \\
& +2\left\{\operatorname{Tr}\left(X_{i_{k}}^{0} X_{j_{k}}^{0}\right)\left(X_{1}^{0}\right)^{\alpha_{1}} \cdots\left(X_{t}^{0}\right)^{\alpha_{t}}\left(X_{i_{k+1}}^{0} X_{j_{k+1}}^{0}\right)\left(X_{t+1}^{0}\right)^{\alpha_{t+1}} \cdots\left(X_{m}^{0}\right)^{\alpha_{m}}\right. \\
& +\operatorname{Tr}\left(X_{i_{k+1}}^{0} X_{j_{k+1}}^{0}\right)\left(X_{1}^{0}\right)^{\alpha_{1}} \cdots\left(X_{t}^{0}\right)^{\alpha_{t}}\left(X_{i_{k}}^{0} X_{j_{k}}^{0}\right)\left(X_{t+1}^{0}\right)^{\alpha_{t+1}} \cdots\left(X_{m}^{0}\right)^{\alpha_{m}} \\
& -\operatorname{Tr}\left(X_{i_{k}}^{0} X_{i_{k+1}}^{0}\right)\left(X_{1}^{0}\right)^{\alpha_{1}} \cdots\left(X_{t}^{0}\right)^{\alpha_{t}}\left(X_{j_{k}}^{0} X_{j_{k+1}}^{0}\right)\left(X_{t+1}^{0}\right)^{\alpha_{t+1}} \cdots\left(X_{m}^{0}\right)^{\alpha_{m}} \\
& -\operatorname{Tr}\left(X_{j_{k+1}}^{0} X_{j_{k}}^{0}\right)\left(X_{1}^{0}\right)^{\alpha_{1}} \cdots\left(X_{t}^{0}\right)^{\alpha_{t}}\left(X_{i_{k}}^{0} X_{i_{k+1}}^{0}\right)\left(X_{t+1}^{0}\right)^{\alpha_{t+1}} \cdots\left(X_{1}^{0}\right)^{\alpha_{m}} \\
& +\operatorname{Tr}\left(X_{i_{k}}^{0} X_{j_{k+1}}^{0}\right)\left(X_{1}^{0}\right)^{\alpha_{1}} \cdots\left(X_{0}^{t}\right)^{\alpha_{t}}\left(X_{j_{k}}^{0} X_{i_{k+1}}^{0}\right)\left(X_{t+1}^{0}\right)^{\alpha_{t+1}} \cdots\left(X_{m}^{0}\right)^{\alpha_{m}} \\
& +\operatorname{Tr}\left(X_{i_{k+1}}^{0} X_{j_{k}}^{0}\right)\left(X_{1}^{0}\right)^{\alpha_{1}} \cdots\left(X_{t}^{0}\right)^{\alpha_{t}}\left(X_{i_{k}}^{0} X_{j_{k+1}}^{0}\right)\left(X_{t+1}^{0}\right)^{\alpha_{t+1}} \cdots\left(X_{m}^{0}\right)^{\alpha_{m}}
\end{aligned}
$$

where $t=j_{k+1}-1$.

Substitute the relation (4.6) into (4.4). Then applying the relation (4.2), we see that the semi-standard monomial $f$ is a linear combination of monomials of the following types:

(1) semi-standard monomials with lexicographically smaller contents than that of $f$, and (2) the monomial

$$
\begin{gathered}
\operatorname{Tr}\left(X_{i_{1}}^{0} X_{j_{1}}^{0}\right) \cdots \operatorname{Tr}\left(X_{i_{k}}^{0} X_{j_{k}}^{0}\right) \operatorname{Tr}\left(X_{i_{k+2}}^{0} X_{j_{k+2}}^{0}\right) \cdots \operatorname{Tr}\left(X_{i}^{0} X_{j}^{0}\right) \\
\times\left(X_{1}\right)^{\alpha_{1}} \cdots\left(X_{t}\right)^{\alpha_{t}} X_{i_{k+1}} X_{j_{k+1}}\left(X_{t+1}\right)^{\alpha_{t+1}} \cdots\left(X_{m}\right)^{\alpha_{m}}
\end{gathered}
$$


Using again (4.2), we make the monomial (4.7) into a semi-standard monomial $g$. Then the content of $g$ is equal to $c(f)$ or lexicographically smaller than $c(f)$. If $c(g)=c(f)$, then one sees immediately that the degree of the trace part of $g$ is smaller than that of $f$. We repeat this process. Then the process terminates within finitely many steps. Therefore any semi-standard monomial is a linear combination of standard monomials. To finish the proof, we have to show that the standard monomials are linearly independent. To do so, we employ the following convention: for given formal power series,

$$
f(t) \leq g(t) \text { means that } a_{i} \leq b_{i} \text { for all } i .
$$

Because any element of $T^{0}(2, m)$ is a linear combination of standard monomials, we have

$$
P\left(T^{0}(2, m), t\right) \leq \sum_{d \geq 0} \sharp\{\text { standard monomials of degree } d\} t^{d} .
$$

Then by (3.1), we obtain

$$
P\left(T^{0}(2, m), t\right) \leq \frac{(1+t)^{m}}{\left(1-t^{2}\right)^{m}} P(K[\mathrm{Gr}(2, m)], t) .
$$

Here the equality holds if and only if the standard monomials are linearly independent. On the other hand, by Proposition 3 and (4.1),

$$
P\left(T^{0}(2, m), t\right)=\frac{1}{(1-t)^{m}} P(K[\mathrm{Gr}(2, m]), t),
$$

and hence the set of standard monomials of $T^{0}(2, m)$ constitutes a $K$-basis of $T^{0}(2, m)$. Then again by (4.1), this completes the proof.

\section{$\S 5$. Proof of Theorem 2}

Let $B^{0}(2, m)$ be the subring of $B(2, m)$ generated by the elements:

$$
\operatorname{Tr}\left(\left(X_{i}^{0}\right)^{2}\right), \quad 1 \leq i \leq m,
$$

and

$$
p_{k}^{0}=\sum_{i+j=k} \operatorname{Tr}\left(X_{i}^{0} X_{j}^{0}\right), \quad 3 \leq k \leq 2 m-1 .
$$

A semi-standard monomial is called an $S$-standard monomial of $T^{0}(2, m)$ if the Young tableau associated with its trace part is $S$-standard.

We now prove by induction on degree that $T^{0}(2, m)$ is a $B^{0}(2, m)$ module generated by the $S$-standard monomials of $T^{\circ}(2, m)$. We assume 
that any element of $T^{0}(2, m)$ with degree $<d$ is a linear combination of $S$-standard monomials over $B^{0}(2, m)$. We then claim that any element of degree $d$ is a linear combination of $S$-standard monomials over $B^{0}(2, m)$. By the induction hypothesis, it is enough to prove our claim for elements of the form

$$
\operatorname{Tr}\left(X_{i_{1}}^{0} X_{j_{1}}^{0}\right) \cdots \operatorname{Tr}\left(X_{i_{r}}^{0} X_{j_{r}}^{0}\right) X_{k_{1}}^{0} \cdots X_{k_{t}}^{0},
$$

with $\quad i_{1}+j_{1} \leq i_{2}+i_{2} \leq \cdots \leq i_{r}+j_{r}, \quad 1 \leq k_{1}<\cdots<k_{t} \leq m$.

Take such an element $f$ and consider the sequence of numbers

$$
\left(i_{1}+j_{1}, \cdots, i_{r}+j_{r}, 2 k_{1}, \cdots, 2 k_{t}\right) \text {. }
$$

Permutating the numbers in the sequence above, we get a sequence of numbers

$$
\left(a_{1}, a_{2}, \cdots, a_{r+t}\right) \text {, with } a_{1} \leq a_{2} \leq \cdots \leq a_{r+t} .
$$

The sequence (5.2) of numbers is called the weight of $f$ (denoted by $w(f)$ ). For example, if

$$
f=\operatorname{Tr}\left(X_{1}^{0} X_{4}^{0}\right) \operatorname{Tr}\left(X_{2}^{0} X_{3}^{0}\right) X_{1}^{0} X_{2}^{0} X_{4}^{0},
$$

we have $w(f)=(2,4,5,5,8)$.

Suppose now that

$$
i_{k}<i_{k+1}<j_{k+1}<j_{k} \quad \text { or } \quad i_{k+1}<i_{k}<j_{k}<j_{k+1} \quad \text { for some } k .
$$

Then by using (4.6) and a similar argument as in the proof of Theorem 1 , it is easily verified that $f$ is a linear combination of monomials with lexikographically smaller weight than $w(f)$. Then clearly the process terminates within finitely many steps and hence any element of $T^{0}(2, m)$ is a $B^{0}(2, m)$-linear combination of standard monomials of the form

$$
\operatorname{Tr}\left(X_{\alpha_{1}}^{0} X_{\beta_{1}}^{0}\right) \cdots \operatorname{Tr}\left(X_{\alpha_{s}}^{0} X_{\beta_{s}}^{0}\right) X_{\gamma_{1}}^{0} \cdots X_{\gamma_{u}}^{0},
$$

with $\quad \alpha_{1} \leq \cdots \leq \alpha_{s}, \quad \beta_{1} \leq \cdots \leq \beta_{s}, \quad 1 \leq \gamma_{1} \leq \cdots \leq \gamma_{u} \leq m$.

Furthermore using the relation

$$
p_{k}^{0}=\sum_{i+j=k} \operatorname{Tr}\left(X_{i}^{0} X_{j}^{0}\right),
$$

and repeating the process used above, we may assume that $\beta_{k}>\alpha_{k}+2$ for all $k, 1 \leq k \leq s$. If $\alpha_{t}=\alpha_{t+1}$ for some $t$, then using the relation (5.3), we replace the factor $\operatorname{Tr}\left(X_{\alpha_{t}}^{0} X_{\beta_{t}}^{0}\right)$ by 


$$
p_{k}^{0}-\sum_{\substack{i+j=k \\ i \neq \alpha_{t}}} \operatorname{Tr}\left(X_{i}^{0} X_{j}^{0}\right), \quad k=\alpha_{t}+\beta_{t},
$$

Similarly if $\beta_{t}=\beta_{t+1}$ for some $t$, we replace the factor $\operatorname{Tr}\left(X_{\alpha_{t+1}}^{0} X_{\beta_{t+1}}^{0}\right)$ by

$$
p_{k}^{0}-\sum_{\substack{i+j=k \\ i \neq \alpha_{t+1}}} \operatorname{Tr}\left(X_{i}^{0} X_{j}^{0}\right), \quad k=\alpha_{t+1}+\beta_{t+1} .
$$

And we repeat the same process as above. Then we finally find that any element of $T^{0}(2, m)$ is a $B^{0}(2, m)$-linear combination of standard monomials of the form

$$
\operatorname{Tr}\left(X_{\alpha_{1}} X_{\beta_{1}}\right) \cdots \operatorname{Tr}\left(X_{\alpha_{s}} X_{\beta_{s}}\right) X_{\gamma_{1}} \cdots X_{r_{u}}
$$

with

$$
\alpha_{1}<\cdots<\alpha_{s}, \quad \beta_{1}<\cdots<\beta_{s}, \quad \gamma_{1}<\cdots<\gamma_{u}
$$

and

$$
\beta_{p}>\alpha_{p}+2 \text { for all } p, 1 \leq p \leq s .
$$

Clearly the condition in (5.5) says that the associated Young tableau

$$
\left[\begin{array}{ccc}
\alpha_{1} & \cdots & \alpha_{s} \\
\beta_{1} & \cdots & \beta_{s}
\end{array}\right]
$$

is $S$-standard. Therefore we have proved that any element of $T^{0}(2, m)$ is a $B^{0}(2, m)$-linear combination of $S$-standard monomials of $T^{0}(2, m)$. Then by (4.1), any element of $T(2, m)$ is a $B(2, m)$-linear combination of standard monomials of $T(2, m)$. Since

$$
P(B(2, m), t)=\frac{1}{(1-t)^{m}\left(1-t^{2}\right)^{3 m-3}},
$$

we, in particular, obtain

$$
P(T(2, m), t) \leq \frac{1}{(1-t)^{m}\left(1-t^{2}\right)^{3 m-3}} \sum_{d \geq 0}\left\{\begin{array}{l}
S \text {-standard mono- } \\
\text { mials of degree } d
\end{array}\right\} t^{d}
$$

By Proposition 2, we have

$$
P(T(2, m), t) \leq \frac{1}{(1-t)^{2 m}} P(K[\operatorname{Gr}(2, m)], t),
$$

where the equality holds if and only if the $S$-standard monomials of $T(2, m)$ are $B(2, m)$-linearly independent. By Procesi's identity, this completes the proof of Theorem 2.

\section{§6. Example}

Consider the Hasse diagram for $\Lambda_{6}$ : 


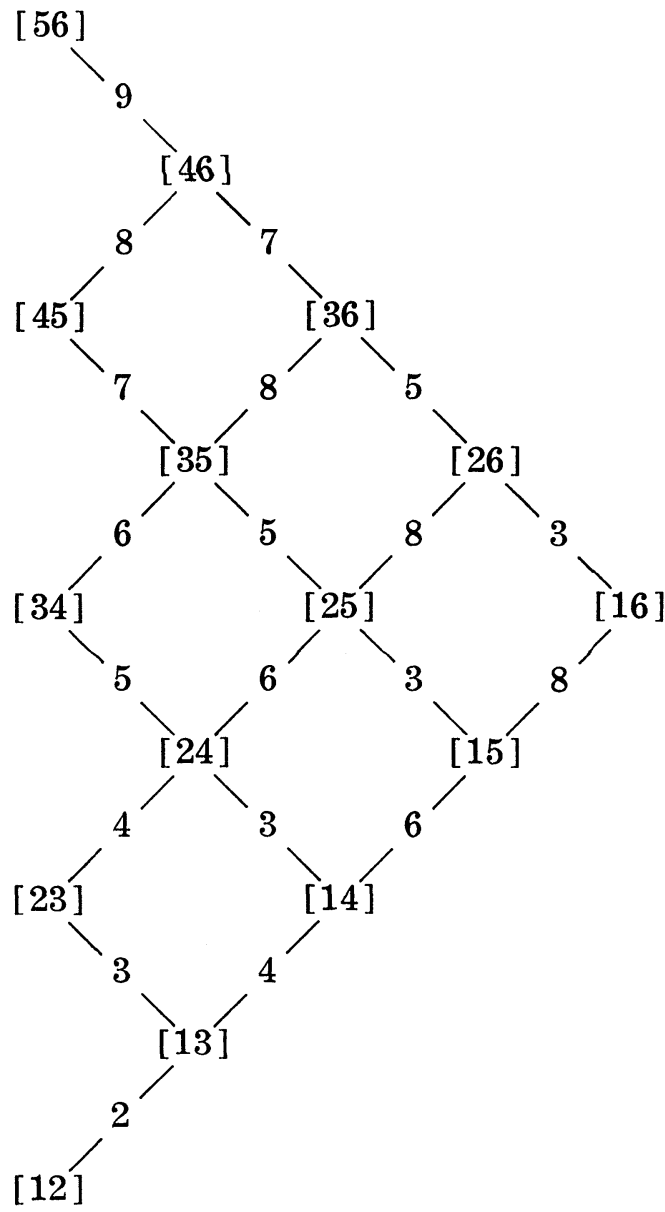

$S$-standard Young tableaux:

$\phi,\left[\begin{array}{l}1 \\ 4\end{array}\right],\left[\begin{array}{l}1 \\ 5\end{array}\right],\left[\begin{array}{l}1 \\ 6\end{array}\right],\left[\begin{array}{l}2 \\ 5\end{array}\right],\left[\begin{array}{l}2 \\ 6\end{array}\right],\left[\begin{array}{l}3 \\ 6\end{array}\right],\left[\begin{array}{ll}1 & 2 \\ 4 & 5\end{array}\right],\left[\begin{array}{ll}1 & 2 \\ 4 & 6\end{array}\right],\left[\begin{array}{ll}1 & 3 \\ 4 & 6\end{array}\right]$, $\left[\begin{array}{ll}1 & 2 \\ 5 & 6\end{array}\right],\left[\begin{array}{ll}1 & 3 \\ 5 & 6\end{array}\right],\left[\begin{array}{ll}2 & 3 \\ 5 & 6\end{array}\right],\left[\begin{array}{lll}1 & 2 & 3 \\ 4 & 5 & 6\end{array}\right]$.

$T(2,6)$ is a free module over the polynomial ring $B(2,6)$ with generators

$$
\operatorname{Tr}(Y) X_{k_{1}} X_{k_{2}} \cdots X_{k_{t}},
$$

where $Y$ is an $S$-standard Young tableau associated with $\Lambda_{6}$ and $X_{k_{1}} X_{k_{2}}$ $\cdots X_{k_{t}}$ is 1 , if $t=0$, or a monic in the generic 2 by 2 matrices

$$
X_{1}, \cdots, X_{6} \text { with } k_{1}<k_{2}<\cdots<k_{t} .
$$




\section{REFERENCES}

[1] L. Le Bruyn, Trace rings of generic 2 by 2 matrices, Mem. Amer. Math. Soc., 363 (1987).

[2] L. Le Bruyn and M. Van den Bergh, An explicit description of $T_{3,2}$, Lecture Notes in Math., 1197, Springer, (1986), 109-113.

[ 3 ] E. Formanek, Invariants and the ring of generic matrices, J. Algebra, 89 (1984), $178-223$.

[4] Y. Teranishi, The Hilbert series of rings of matrix concomitants, Nagoya Math. J., 111 (1988), 143-156.

[5] C. Procesi, Computing with 2 by 2 matrices, J. Algebra, 87 (1984), 342-359.

[6 ] W. Hodge and D. Pedoe, Methods of Algebraic Geometry, vol 2, Cambridge, 1968.

[7] A. Garsia, Combinatorial methods in the theory of Cohen-Macaulay rings, Adv. in Math., 38 (1980), 229-266.

Department of Mathematics

School of Science

Nagoya University

Chikusa.ku, Nagoya 464-01

Japan 